\title{
Spaced transmitter measurements of medium scale traveling ionospheric disturbances near the equator
}

\author{
John MacDougall, ${ }^{1}$ M. A. Abdu, ${ }^{2}$ Inez Batista, ${ }^{2}$ Ricardo Buriti, ${ }^{3}$ A. F. Medeiros, ${ }^{3}$ \\ P. T. Jayachandran, ${ }^{4}$ and Gilvan Borba ${ }^{5}$ \\ Received 20 June 2011; revised 21 July 2011; accepted 24 July 2011; published 26 August 2011.
}

[1] The motion of Medium Scale Traveling Ionospheric Disturbances (MSTIDs) was measured for 1 year from the eastern tip of Brazil, about $10^{\circ}$ degrees south of the magnetic and geographic equators using spaced fixed frequency radio transmitters. The motion was very similar during all of the year. The daytime average direction was towards $150^{\circ}$ azimuth (clockwise from north) and speed was typically in the range $150-300 \mathrm{~m} / \mathrm{s}$. The azimuth varied only slightly during the day being slightly more easterly during evening-morning hours. During winter months there were also some TIDs whose motion was northward. None of the known sources for MSTIDs fits well with MSTIDs having these velocity patterns. A possible unconfirmed source is the Inter-Tropical Convergence Zone (ITCZ). Citation: MacDougall, J., M. A. Abdu, I. Batista, R. Buriti, A. F. Medeiros, P. T. Jayachandran, and G. Borba (2011), Spaced transmitter measurements of medium scale traveling ionospheric disturbances near the equator, Geophys. Res. Lett., 38, L16806, doi:10.1029/2011GL048598.

\section{Introduction}

[2] There have been few systematic measurements of Medium Scale Traveling Ionospheric Disturbances (MSTID) near the equator. However there is a particular interest in MSTIDs in this region because of their implied role [Miller et al., 1997] in the triggering of plasma bubbles and associated strong Spread-F irregularities that can cause dropouts and scintillation of GPS based systems [Kintner et al., 2007]. At mid latitudes the properties of MSTIDs are reasonably well known because of many short and also longer time sets of measurements [Munro, 1958; Waldock and Jones, 1986; MacDougall et al., 2009a]. These studies showed that there is considerable variation in the propagation of MSTIDs as a function of time-of-day and season. Briefly the typical daytime propagation direction is eastwards with a tendency to gradually rotate towards a more equatorward direction during the day. During nighttime the typical propagation direction becomes westward-equatorward.

\footnotetext{
${ }^{1}$ Department of Electrical and Computer Engineering, University of Western Ontario, London, Ontario, Canada.

${ }^{2}$ INPE, Sao Jose dos Campos, Brazil.

${ }^{3}$ Department of Physics, Universidade Federal de Campina Grande, Campina Grande, Brazil.

${ }^{4}$ Department of Physics, University of New Brunswick, Fredericton, New Brunswick, Canada.

${ }^{5}$ Department of Geophysics, Universidade Federal do Rio Grande do Norte, Natal, Brazil.

Copyright 2011 by the American Geophysical Union. 0094-8276/11/2011GL048598
}

[3] Near the equator there are a few TID studies. The measurements by Rottger [1977] in Peru, using a technique similar to that used for the present study, showed most TIDs propagating approximately southward. In the western longitudes of South America a recent study of TIDs by Makela et al. [2010] used optical methods and so has data only for nighttime TIDs. They found that their TIDs propagated towards the northwest. Another optical study of MSTIDs by Shiokawa et al. [2006] in Indonesia at geomagnetic latitude $-10.4^{\circ}$ found southward propagating TIDs. A study of MSTIDs in Malaysia by Husin et al. [2011] based on GPS total electron content measurements showed south-westward propagating MSTIDs during both day and night. The measurement locations in Malaysia (they used several GPS receiver sites) were around $3^{\circ}$ north of the geographic equator or about $6^{\circ}$ south of the magnetic equator. To summarise these equatorial TID measurements in both hemispheres, apart from Makela et al. [2010], the TID propagation directions tend to be southward and slightly westward.

[4] In this paper we are reporting on one year of continuous MSTID measurements at a location a few degrees south of the geographic and magnetic equators in Brazil.

\section{Measurements}

\subsection{Experimental Setup}

[5] These measurements use the method of radio ionospheric reflection from spaced transmitters for measuring the MSTID motion. The basic measurement technique is similar to that described by MacDougall et al. [2009a], with a few differences as detailed below. The Brazil setup uses the 3 sites Caico, Cariri and Campina Grande shown on the Figure 1 map. At Caico and Cariri are 30W Legendre coded transmitters, and at Campina Grande is a standard $600 \mathrm{~W}$ CADI ionosonde that is used for both transmitting and receiving. By analyzing the same TID seen both overhead at Campina Grande and at the midpoints of the CaicoCampina Grande and Cariri-Campina Grande paths one can determine time delays and hence the TID velocity. Unfortunately, the triangle of station locations is far from ideal. An ideal triangle for these stations would be to have Campina Grande at the apex of a right angled triangle with equal sides of length about $100 \mathrm{~km}$ to the two remote transmitters. The arrangement of sites was based on the availability of suitable places for equipment operation, so it was not possible to locate the sites in an ideal geometry.

[6] An interesting technical detail is that the measurement of the TID motion uses different pulse coding to separate the pulses coming from the Campina Grande CADI and the remote $30 \mathrm{~W}$ transmitters. The first pulse sequence is triggered by the $1 \mathrm{sec}$ time pulse from the GPS satellite receiver. 


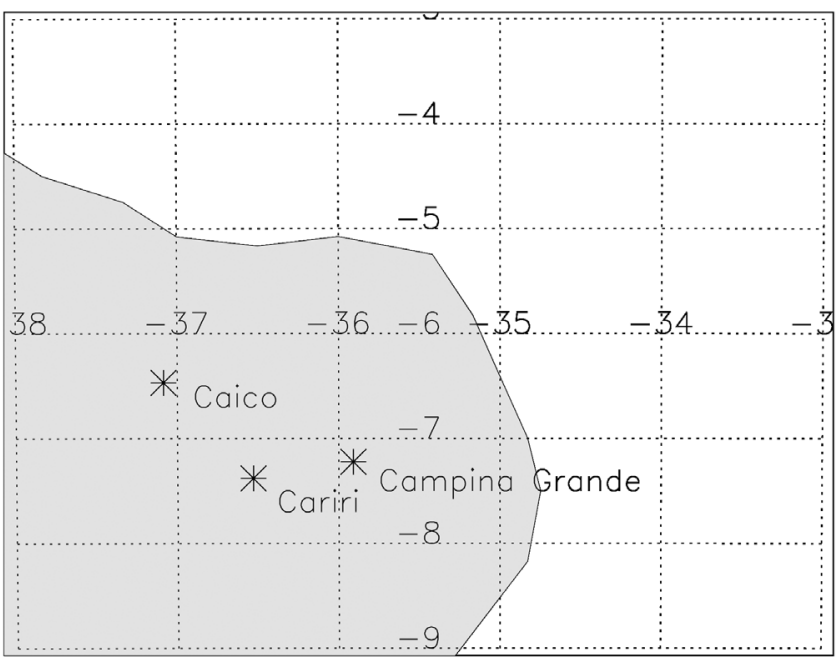

Figure 1. Location of the 3 stations in the east most part of Brazil. Geographic latitude and longitude coordinates are marked on the figure. The magnetic equator is located at approximately $+4^{\circ}$ latitude for these longitudes.
At this time the Cariri remote transmitter sends a 113 bit Legendre coded pulse and the Campina Grande CADI sends an 8 bit complimentary coded pulse. Both are received by the Campina Grande CADI. Then $1 / 20 \mathrm{sec}$ later the other remote transmitter at Caico sends a Legendre pulse and the Campina Grande CADI sends the 2nd 8 bit complimentary sequence pulse. Because the complimentary and Legendre pulses are essentially uncorrelated it is possible to distinguish both the overhead echo from Campina Grande and the echoes from the 2 remote transmitters by correlating the received echoes with the different codes. The basic advantage of this scheme is that it reduces the size of the raw data files since the 3 station measurements are obtained from the data for 2 pulses.

[7] For the Brazil measurements a fixed frequency of $5 \mathrm{MHz}$ was used. While this frequency gave quasi-continuous daytime echoes, usually during nighttime the critical frequency fell below $5 \mathrm{MHz}$ so there was a data gap of a few hours.

[8] Station pairs of the recorded data after decoding was visually displayed and an operator could time shift the traces so as to determine the best delay between the pairs of stations. Figure 2 is a 'screen shot' of the analysis for a typical

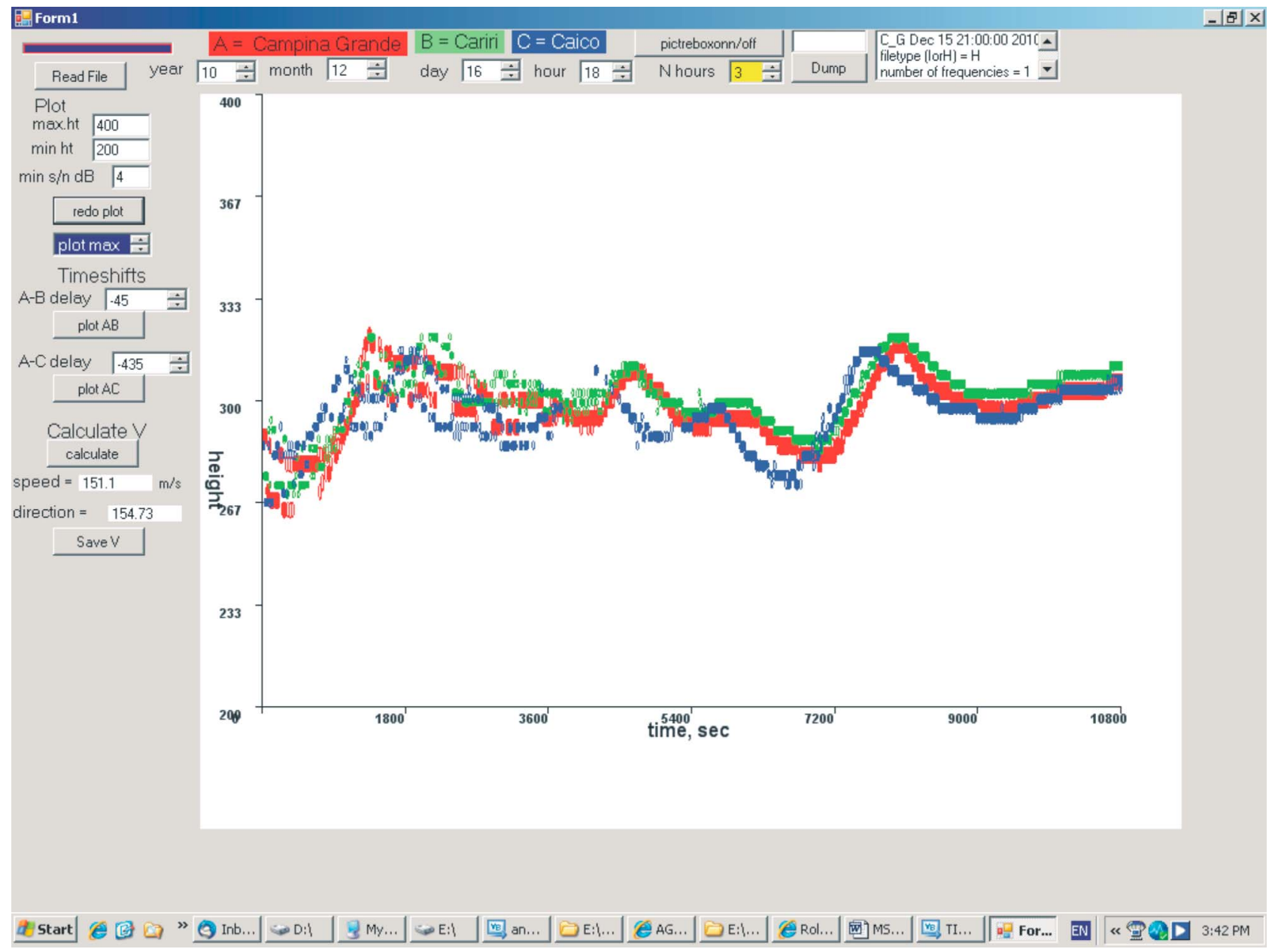

Figure 2. Screenshot of the TID analysis. The traces from the 3 stations can be seen in colour (see top of figure for station identification). For this plot the option of just plotting the height of maximum signal amplitude for each time has been chosen to make the plot clearer. When doing the analysis the stations in pairs are replotted with timeshifts and the timeshifts are adjusted for the best fits. The numbers in the 'delay' boxes show the best fit values, in seconds, determined for this sample. 

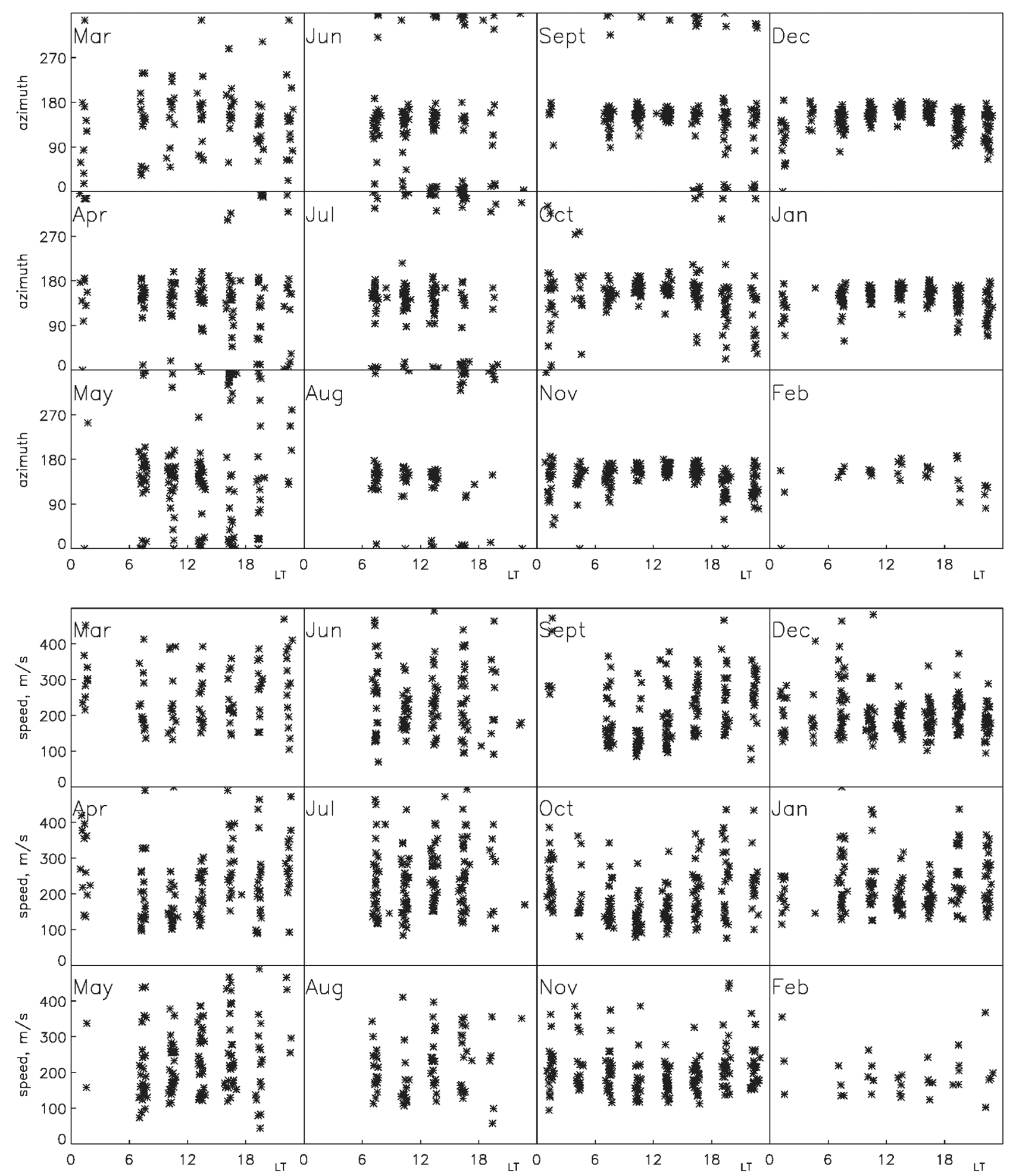

Figure 3. (top) Azimuth and (bottom) speed of measured TID velocities. Months are from March 2010 to February 2011. Measurements were done in 3 hour data segments, hence the clustering of data points every 3 hours. When plotted a random time was added to each of the data points to make the data distribution easier to see.

3 hour interval. We tried numerically determining the timeshifts using correlation, but the 'eyeball' determination of timeshift gave better results and was used for all the measurements presented here. A comment on the 'eyeball' determination of timeshifts is that it is easiest to determine the timeshifts between features that have short periods so there may be some biasing of the results in favour of short period TIDs.

\subsection{Experimental Results}

[9] The TID measurements started in March 2010 and

Figure 3 shows the results for a period of one year of the 


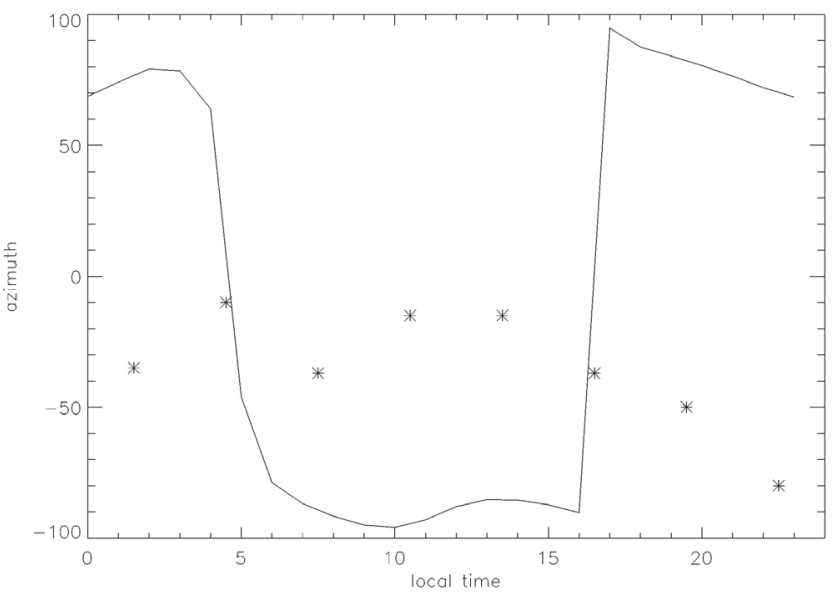

Figure 4. Wind at $300 \mathrm{~km}$ height over Campina Grande for 2010 October from the HWM model (solid curve) and mean measured TID propagation directions (reversed) for this same month.

distribution by month of the MSTID propagation directions and speed. The start month (March 2010) and the end month (Feb. 2011) have data for only a small number of days. Other months have data for all days but there are often missing TID velocities at night and when there was strong sporadic-E and spread F occurrences.

[10] We found that there was always some TID activity present, although at times the TID height variations were small. Unfortunately observing TIDs by the variations in the virtual height that the Gravity Waves produce does not allow one to easily determine the relative size of the gravity waves since the observed height variations depend on the height gradient of the electron density [see MacDougall et al., 2009b]. Therefore a more elaborate analysis involving 'true height' analysis of collocated ionograms is necessary to obtain useful measurements about wave amplitudes, and hence wave periods, from virtual height variation measurements. Such an analysis was not available for these Brazil measurements.

[11] The main characteristics of the TID velocities shown in Figure 3 may be summarized as follows.

[12] Azimuths with respect to north of the TID velocity (Figure 3, top):

[13] 1. The pattern for most months is similar with directions $\sim 150^{\circ}$ for midday and a tendency for slightly smaller azimuths in early and late hours. There is no obvious switch of the azimuth directions during nighttime that is seen for midlatitude TID measurements.

[14] 2. For months April-September there are azimuths near $0^{\circ}$ in afternoon-evening on some days.

[15] 3. There is a clear absence of westward velocities throughout the year (with some minor exceptions).

[16] Speed of the TIDs (Figure 3, bottom):

[17] 4. The pattern of speeds is the same in all months. Speed tends to be lowest around midday, possibly because the reflection heights at the $5 \mathrm{MHz}$ fixed frequency are lower at this time. Speeds are similar to those measured in midlatitudes [MacDougall et al., 2009a].
[18] There is a considerable scatter of speeds, possibly because the TIDs have a range of periods. Most speeds are between approximately 150 and $300 \mathrm{~m} / \mathrm{s}$.

\section{Discussion}

[19] These TID velocities clearly have a different pattern of propagation azimuths than what is observed at midlatitudes. The dominant azimuth of TID propagation here is $\sim 150^{\circ}$, with, during local winter months (Apr.-Sept.), some azimuths near $0^{\circ}$. Thus the indicated source locations with respect to Campina Grande would be in the $-30^{\circ}$ and $180^{\circ}$ azimuth directions. One can see from Figure 1 that the source positions for these two directions would be over ocean areas. Of course detailed raytracing will slightly move the source locations from being simply located along the reverse of the propagation directions [Kubota et al., 2011]. Originally it was expected that the well known atmospheric disturbances in the Amazon region would show up as the source of TIDs for Brazil. However, neither the $-30^{\circ}$ or $180^{\circ}$ source directions is near to the Amazon region direction which is $\sim 280^{\circ}$ (or $\sim 80^{\circ}$ ) with respect to Campina Grande. Our measured propagation directions for the TIDs are in reasonable agreement with those reported by Rottger [1977] and Shiokawa et al. [2006].

[20] We continue this discussion by looking at a number of possible TID sources to see if any of them are compatible with our TID observations. In general we will find that none of possible sources matches well with our observations. We examine here a number of possible TID sources starting with known sources of TIDs at midlatitudes. At midlatitudes there are 3 known sources: (1) the sunrise/sunset terminators, (2) gravity waves propagating up from the troposphere, (3) auroral magnetic disturbances [MacDougall and Jayachandran, 2011].

\subsection{The Sunrise/Sunset Terminators as the Source}

[21] For more details on this source see [MacDougall and Jayachandran, 2011, and references therein]. At midlatitude this produces approximately eastward propagating TIDs after sunrise, and approximately westward propagating waves after sunset [MacDougall et al., 2009a]. Our measured Brazil TID propagation directions are incompatible with these propagation directions and it therefore appears that the terminator is not the source for our Brazil TIDs.

\subsection{Upward Propagating Tropospheric Gravity Waves}

[22] Looking at the possible source of gravity waves propagating upward from the troposphere, we present in Figure 4 the average reversed measured TID propagation direction (* points), and the $300 \mathrm{~km}$ wind from the HWM model [Hedin et al., 1996]. Due to critical effects TIDs propagating in the wind direction can get removed and only the TIDs propagating in the opposite direction to the wind will be left. At midlatitudes there is good agreement between the wind and the reversed TID propagation directions [Waldock and Jones, 1986; MacDougall et al., 2009a]. However in Brazil, as can be seen from Figure 4, there is no agreement between reversed TID propagation direction and the $\mathrm{F}$ region wind direction. 

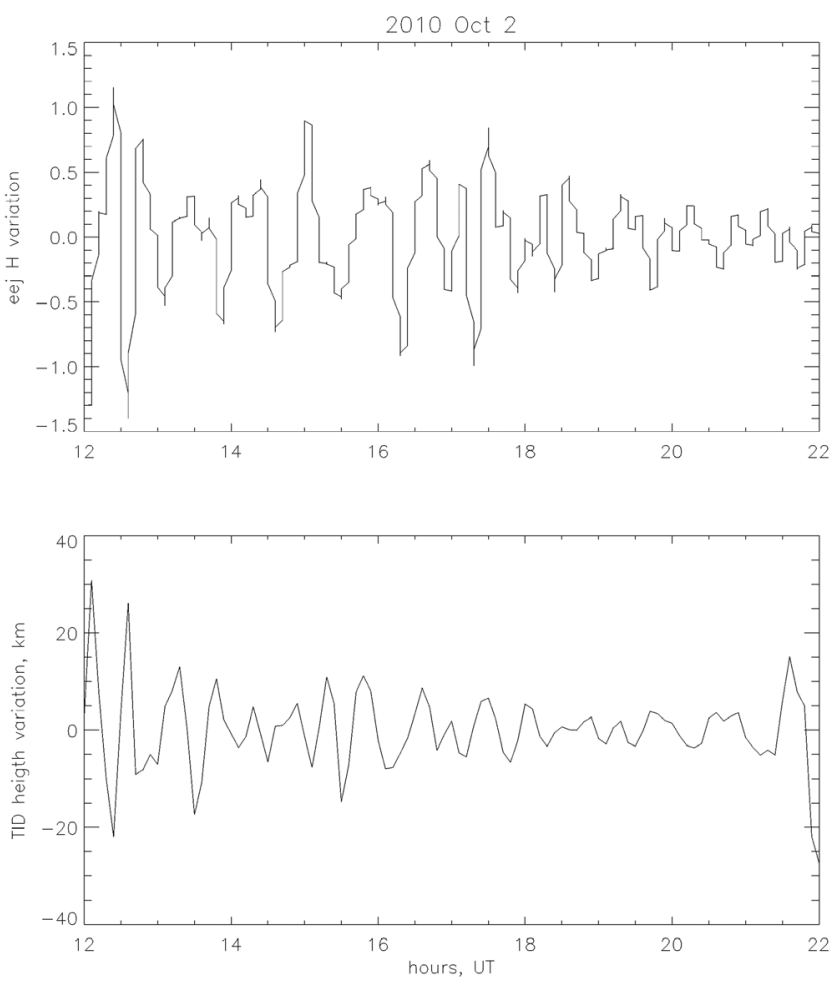

Figure 5. Filtered EEJ variations and TID height variations for 2010 October 2. Filter bandpass 15-90 minutes.

\subsection{Auroral Disturbance as Possible Source}

[23] In this respect we note that for the $\sim 150^{\circ}$ propagating TIDs the correlation of wave amplitudes (just using RMS values for the size of the virtual height fluctuations) and $\mathrm{Kp}$ index was very close to zero.

[24] Therefore none of the 3 midlatitude TID sources appears to be the source for these Brazil TIDs so we next look at other possible TID sources. One possible source that is compatible with the $150^{\circ}$ propagation direction (or $-30^{\circ}$ source direction) would be associated with the equatorial electrojet (EEJ) since the tilt of the magnetic equator is close to $-30^{\circ}$ in this region. It is well known that TIDs are generated by the auroral electrojet [Hunsucker, 1982]. The same processes that generate TIDs at the auroral electrojet could operate at the EEJ.

[25] To examine a possible EEJ source for the TIDs, magnetometer data was obtained for station Tatuoca $\left(-12^{\prime}\right.$ latitude, $-4830^{\prime}$ longitude, $0.44^{\circ} \mathrm{mag}$. lat.) which is located at the mouth of the Amazon River westward of these TID observations. Ideally, to rigorously separate the EEJ variations, one needs both an EEJ station (Tatuoca) and a station just outside the EEJ. However as a first approximation just the Tatuoca horizontal magnetometer variations are used. The TID height variations for Campina Grande and the Tatuoca magnetic variation were bandpass filtered to select periods between 15 and 90 minutes (typical TID periods). Then the standard deviations of these two variations were calculated for 10 daytime hours (12-22 UT hours) and compared. The cross correlation for the two months September-October 2010 was negligible, being -0.06 .

[26] Figure 5 shows a comparison of EEJ and TID variations for a typical day. The two variations look similar but are, in fact, poorly correlated. Further reasons for rejecting the EEJ as a source are: On most days the magnitude of the TID variations was about the same whereas the EEJ variations were negligible on some days. Also the auroral electrojet variations that generate TIDs are much larger than these EEJ variations. Finally the EEJ variations show a definite correlation with the Kp index while the TID variations had a negligible correlation with the Kp. In summary therefore there does not seem to be any evidence that the source for these Brazil TIDs is in any way associated with the EEJ.

[27] A final suggestion for the source of the $150^{\circ}$ propagating TIDs is the Inter-Tropical Convergence Zone (ITCZ). This has previously been suggested as a source for TIDs [Rottger, 1977; Tsunoda, 2010]. The ITCZ is easily seen in weather satellite pictures and shows as a persistent relatively narrow band of clouds near the geographic equator. The orientation of the ITCZ in the Brazil-Atlantic sector is usually approximately east-west [Waliser and Gautier, 1993] and for the Western Atlantic region the ITCZ has an approximately $10^{\circ}$ annual migration in latitude being furthest south in December solstice and furthest north in June solstice [Citeau et al., 1986; Waliser and Gautier, 1993; Tsunoda, 2010]. In all months the average position of the ITCZ for the Atlantic near Brazil is north of the geographic equator which is therefore compatible with it being a possible source for the $150^{\circ}$ propagating TIDs. Unfortunately it is not possible to prove that the ITCZ is the source for the TID since the only observed property of the ITCZ appears to be its location in latitude. To obtain further evidence for a connection between the ITCZ and TIDs a repeat of the TID measurements at a location where the ITCZ is, for instance, located south of the measurement location should show northward propagating TIDs if the ITCZ is the source region.

[28] The above discussion has looked mainly at the $150^{\circ}$ propagating TIDs. In months from April to September some TIDs propagating at approximately $0^{\circ}$ are observed. TIDs propagating in this direction are inconsistent with almost all of the possible sources mentioned above, including the ITCZ. Of course there is a possibility that these $0^{\circ}$ propagating TIDs are coming from auroral disturbances so we looked at the correlation of the number of $3 \mathrm{hr}$ intervals that had northward propagating TIDs on each day of June and July and the sum $\mathrm{Kp}$ on these days. The correlation was 0.08 which seems to also eliminate auroral and other magnetic disturbances as a possible source for this group of TIDs. A further source possibility for these northward propagating TIDs might be the known TIDs that are generated by powerful weather disturbances near the Antarctic continent [Galushko et al., 2007]. Another TID study that infers waves from a source located in an ocean area is the Puerto Rico incoherent radar study of TIDs by Djuth et al. [2010].

\section{Conclusions}

[29] Measured propagation velocities of TIDs in eastern Brazil show a quite consistent pattern. Speeds range from $150-300 \mathrm{~m} / \mathrm{s}$. Midday directions are mostly around $150^{\circ}$ azimuth with a small daily variation and there is a second group of TIDs propagating near northward in April to September months. The known sources of TIDs at midlatitudes do not appear to be the sources of either the $150^{\circ}$ or 
the $0^{\circ}$ propagating TIDs. A possible source for the $150^{\circ}$ TIDs is the ITCZ.

[30] Acknowledgments. This research was supported by grants from the National Science and Engineering Research Council of Canada. Development of the TID transmitters was supported by the Canadian Space Agency. The Tatuoca magnetometer data was kindly provided by Sergio Fontes, from the National Observatory, Rio de Janeiro.

[31] The Editor thanks two anonymous reviewers for their assistance in evaluating this paper.

\section{References}

Citeau, J., L. Finaud, J. P. Cammas, and Y. Gouriou (1986), Questions about the ITCZ migration over the tropical Atlantic sea surface temperature in the Gulf of Guinea and the runoff of Senegal river, in Proceedings of the 6th METEOSAT Scientific User's Meeting, pp. 1-17, Eumetsat, Darmstadt-Eberstadt, Germany.

Djuth, F. T., L. D. Zhang, D. J. Livneh, I. Seker, S. M. Smith, M. P. Sulzer, J. D. Mathews, and R. L. Walterscheid (2010), Arecibo's thermospheric gravity waves and the case for an ocean source, J. Geophys. Res., 115, A08305, doi:10.1029/2009JA014799.

Galushko, V. G., A. S. Kashcheyev, S. B. Kashcheyev, A. V. Koloskov, I. I. Pikulik, Y. M. Yampolski, V. A. Litvinov, G. P. Milinevsky, and S. Rakusa-Suszczewski (2007), Bistatic HF diagnostics of TIDs over the Antarctic Peninsula, J. Atmos. Sol. Terr. Phys., 69, 403-410, doi:10.1016/j.jastp.2006.05.010.

Hedin, A. E., et al. (1996), Empirical wind model for the upper, middle and lower atmosphere, J. Atmos. Sol. Terr. Phys., 58, 1421-1447, doi:10.1016/0021-9169(95)00122-0.

Hunsucker, R. A. (1982), Atmospheric gravity waves generated in the high-latitude ionosphere: A review, Rev. Geophys., 20, 293-315, doi:10.1029/RG020i002p00293.

Husin, A., M. Abdullah, and M. A. Momani (2011), Observation of mediumscale traveling ionospheric disturbances over Peninsular Malaysia based on IPP trajectories, Radio Sci., 46, RS2018, doi:10.1029/2010RS004408.

Kintner, P. M., B. M. Ledvina, and E. R. de Paula (2007), GPS and ionospheric scintillations, Space Weather, 5, S09003, doi:10.1029/ 2006SW000260.

Kubota, M., M. Conde, M. Ishii, Y. Murayama, and H. Jin (2011), Characteristics of nighttime medium scale traveling ionospheric disturbances observed over Alaska, J. Geophys. Res., 116, A05307, doi:10.1029/ 2010JA016212.

MacDougall, J. W., and P. T. Jayachandran (2011), Solar terminator and auroral sources for traveling ionospheric disturbances in the midlatitude F region, J. Atmos. Sol. Terr. Phys., in press.
MacDougall, J., G. Li, and P. T. Jayachandran (2009a), Traveling ionospheric disturbances near London, Canada, J. Atmos. Sol. Terr. Phys., 71, 2077-2084, doi:10.1016/j.jastp.2009.09.016.

MacDougall, J., M. Abdu, I. Batista, P. R. Fagundes, Y. Sahai, and P. T. Jayachandran (2009b), On the production of traveling ionospheric disturbances by atmospheric gravity waves, J. Atmos. Sol. Terr. Phys., 71, 2013-2016, doi:10.1016/j.jastp.2009.09.006.

Makela, J. J., E. S. Miller, and E. R. Talaat (2010), Nighttime mediumscale traveling ionospheric disturbances at low geomagnetic latitudes, Geophys. Res. Lett., 37, L24104, doi:10.1029/2010GL045922.

Miller, C. A., W. E. Swartz, M. C. Kelley, M. Mendillo, D. Nottingham, J. Scali, and B. Reinish (1997), Electrodynamics of midlatitude spread F: 1. Observations of unstable gravity wave-induced ionospheric electric fields at tropical latitudes, J. Geophys. Res., 102, 11,521-11,532, doi:10.1029/96JA03839.

Munro, G. H. (1958), Travelling ionospheric disturbances in the F region, Aust. J. Phys., 11, 91-113, doi:10.1071/PH580091.

Rottger, J. (1977), Travelling disturbances in the equatorial ionosphere and their association with penetrative cumulus convection, J. Atmos. Terr. Phys., 39, 987-998, doi:10.1016/0021-9169(77)90007-1.

Shiokawa, K., Y. Otsuka, and T. Ogawa (2006), Quasiperiodic southward moving waves in 630-nm airglow images in the equatorial thermosphere, J. Geophys. Res., 111, A06301, doi:10.1029/2005JA011406.

Tsunoda, R. T. (2010), On seeding equatorial spread F during solstices, Geophys. Res. Lett., 37, L05102, doi:10.1029/2010GL042576.

Waldock, J. A., and T. B. Jones (1986), HF Doppler observations of medium-scale traveling ionospheric disturbances at mid-latitudes, J. Atmos. Terr. Phys., 48, 245-360, doi:10.1016/0021-9169(86)90099-1.

Waliser, D. E., and C. Gautier (1993), A satellite-derived climatology of the ITCZ, J. Clim., 6, 2162-2174, doi:10.1175/1520-0442(1993) $006<2162:$ ASDCOT $>2.0$.CO;2.

M. A. Abdu and I. Batista, INPE, Ave. dos Astronautas 1758, Sao Jose dos Campos, SP 12245-970, Brazil.

G. Borba, Department of Geophysics, Universidade Federal do Rio Grande do Norte, Caixa Postal 1524, Natal, CEP 59072-970, Brazil.

R. Buriti and A. F. Medeiros, Department of Physics, Universidade Federal de Campina Grande, Rua Aprigio Veloso 882, Bairro Universitario, Campina Grande, CEP 58429-140, Brazil.

P. T. Jayachandran, Department of Physics, University of New Brunswick, PO Box 4400, Fredericton, NB E3B 5A3, Canada.

J. MacDougall, Department of Electrical and Computer Engineering, University of Western Ontario, 1155 Richmond St., London, ON N6A 5B9, Canada. (jmacdoug@uwo.ca) 